Meta

Journal des tradlucteurs

Translators' Journal

\title{
Réflexions sur le langage du droit : problèmes de langue et de style
}

\section{Jean-Claude Gémar}

Volume 26, numéro 4, décembre 1981

URI : https://id.erudit.org/iderudit/002846ar

DOI : https://doi.org/10.7202/002846ar

Aller au sommaire du numéro

Éditeur(s)

Les Presses de l'Université de Montréal

ISSN

0026-0452 (imprimé)

1492-1421 (numérique)

Découvrir la revue

Citer cet article

Gémar, J.-C. (1981). Réflexions sur le langage du droit : problèmes de langue et de style. Meta, 26(4), 338-349. https://doi.org/10.7202/002846ar d'utilisation que vous pouvez consulter en ligne.

https://apropos.erudit.org/fr/usagers/politique-dutilisation/ 


\section{Réflexions sur le langage du droit: problèmes de langue et de style*}

JeAN-Claude Gémar

Le langage du droit a toujours exercé un étrange pouvoir de fascination sur les linguistes, mais, dans le même temps, il semble les tenir à distance, les exclure d'un cercle réservé aux initiés : «Non-juristes, s'abstenir» ou «Nul n'entre ici s'il n'est juriste».

Car comment expliquer autrement le peu de progrès réalisés dans un domaine qui incarne pourtant l'expression du fait social, de la norme imposée à tous par le biais de la règle juridique? Les juristes ne laissent à personne le soin d'analyser et d'interpréter le langage du droit dont ils seraient les seuls dépositaires : l'interprétation des textes de loi leur revient de droit. Que je sache, cette fonction n'est jamais confiée à des non-juristes, elle est l'apanage du juge et l'on voit mal comment un profane, fût-il sémanticien, pourrait se voir confier le soin d'intervenir dans un domaine traditionnellement "réservé». Nous touchons là aux prérogatives de l'État et non plus aux simples intérêts publics ou privés. L'intérêt général bien compris passe par le canal de la puissance publique, ce qui met en cause le principe de la «légitimité», que je n'aborderai pas ici.

Pour toutes ces raisons, et pour d'autres encore ${ }^{1}$, on pourrait penser que la linguistique est appelée à jouer un rôle marginal auprès des sciences juridiques (tant en phonologie qu'en morphologie, en lexicologie qu'en sémantique ou en stylistique), comme semblent le penser certains linguistes (dont Mounin) qui ne voient dans le langage du droit qu'un mode d'expression (linguistique) d'un message (juridique).

De fait, les progrès de la linguistique générale, sauf en de rares exceptions, n'ont guère influencé l'étude des diverses formes que peut revêtir le discours juridique. Pourtant, à l'expérience, il semble bien que le traducteur, entre autres, aurait beaucoup à apprendre d'une «jurilinguistique» dont l'objet serait l'étude systématique, diachronique et synchronique, du langage du droit, notamment sur le plan stylistique puisque la rédaction joue un rôle essentiel en traduction. Mais il va de soi que le traducteur ne serait qu'un des nombreux spécialistes visés par une jurilinguistique qui, bénéficiant de l'apport de la lexicologie et axée sur les aspects sémantiques du langage du droit, permettrait de dégager, par exemple, un vocabulaire fondamental du droit ${ }^{2}$, ou, par le

Colloque de Gendon, 1980.

1. Georges Mounin (1979): «La linguistique comme science auxiliaire dans les disciplines juridiques », Meta, numéro spécial sur La traduction juridique, vol. 24, $\mathrm{n}^{\circ} 1$, p. 9-17.

2. VOFOD: projet de recherche de l'auteur en vue d'établir un vocabulaire fondamental du droit canadien. 
biais d'une analyse morphosyntaxique de ce même langage, de relever par exemple, les difficultés inhérentes au langage du droit dans le contexte d'un Canada bilingue ${ }^{3}$.

Dans la plupart des pays de common law et de droit civil, un nombre croissant de juristes s'interrogent sur l'énoncé linguistique de la règle juridique. Ils sont poussés par de nombreuses raisons qui, si elles ne s'inspirent pas toutes de préoccupations sociales, n'en témoignent pas moins d'un grand intérêt pour un genre de communication privilégiée, qui s'adresse au plus grand nombre lorsqu'elle revêt la forme collective et générale de la Loi. Communication? Oui, unilatérale, formelle, impérative, émanant de l'autorité supérieure et imposée en amont à l'ensemble de la collectivité qui doit s'y conformer sous peine de sanction. Sans doute. Mais communication quand même!

En s'attaquant sans complexe au vieux problème que pose la formulation d'un message destiné au grand public, ces juristes font œuvre de linguistes. Ces jurilinguistes, puisqu'il faut bien les appeler par leur nom, qu'ils soient juristes et linguistes ou linguistes et juristes de formation, s'efforcent de rendre accessible le langage du droit à l'homme de la rue, de le démythifier en le ramenant à une forme d'expression optimale qui répondrait à l'idéal de concision, de clarté et de simplicité vers lequel il devrait tendre. Les recherches des jurilinguistes nord-américains présentent sur ce plan un très grand intérêt, tout particulièrement pour le traducteur, principal bénéficiaire et première «victime» des réusșites et des échecs des jurilinguistes.

C'est en effet le traducteur qui sert parfois de cobaye aux expériences langagières des uns et des autres, aussi n'est-il pas étonnant qu'il ait pris le parti d'intervenir dans le débat et d'y faire entendre une voix autorisée par de longues heures de lutte quotidienne contre les difficultés, les pièges et les ambiguïtés que lui réserve l'opération de transfert d'un système juridique à un autre.

Prenons le cas du traducteur œuvrant dans le contexte du Canada, et plus particulièrement au Québec.

\section{Le Québec : un cas particulier}

Voici un pays de langue française dont la plupart des institutions ont été créées sur un modèle anglais. Aussi ne peut-on enseigner au Québec les langues de spécialité que sont les langues commerciale et juridique, par exemple, en se fondant sur des notions, des sources et une terminologie essentiellement «hexagonales». Le français «international» lui-même se révèle impuissant lorsqu'il faut rendre, dans un contexte socio-politique différent, des notions émanant d'institutions et d'organes inconnus d'un autre système, dont la valeur normative, en raison d'un accident historique, se fonde sur le droit anglais ${ }^{4}$. Toute approche de la traduction, du moins dans le domaine des sciences sociales, doit tenir compte de ce facteur.

3. Projet de recherche sur les difficultés de la langue juridique française au Canada, sous la direction de l'auteur.

4. Voir Henri Brun et Guy Tremblay (1972) : «Les origines historiques du droit public canadien», dans Droit public fondamental, Québec, P.U.L., p. 11-12. 


\section{Langue technique et langue des sciences sociales}

Dès lors, il faut faire une distinction fondamentale entre les disciplines ressortissant aux sciences sociales ${ }^{5}$ et les matières proprement "scientifiques" parce que celles-là font intervenir des méthodes, des techniques, des concepts et des préoccupations différents et, parfois, opposés. Les langues «techniques» sont probablement les seules dont on puisse retenir, aux fins d'enseignement, le fonds terminologique, généralement univoque quels que soient le lieu et la langue employés. Il n'en va pas de même pour les sciences sociales, polysémiques par définition puisque leur discours s'appuie non sur des relations objectives vérifiables mais sur l'étude de l'activité humaine, variable à l'infini. On voit d'emblée quels obstacles épistémologiques s'opposent à l'établissement de leur statut scientifique et les difficultés d'ordre méthodologique que présente leur enseignement.

Avant d'aborder certains aspects linguistiques de la langue de spécialité juridique, je vais essayer de cerner d'un peu plus près la notion de langue de spécialité appliquée à la langue du droit.

\section{Économie générale de $L S$}

Les théoriciens du langage sont loin d'être d'accord sur une définition de la langue de spécialité, qui est fonction de la matière enseignée. Les efforts de recherche collectifs ont essentiellement porté sur la définition des langues de spécialité techniques, dont la terminologie est moins polysémique, moins sujette à interprétation que celle des sciences sociales.

Toute tentative de définition de la langue de spécialité se heurte inévitablement, dès l'abord, aux problèmes de terminologie et de sémantique posés par les divers synonymes de "langue courante ${ }^{6}$, expression qu'il est nécessaire d'opposer à langue de spécialité. En outre, le vocabulaire fondamental de la langue courante et le vocabulaire spécialisé contiennent de nombreux termes que l'on peut attribuer indifféremment à l'un ou à l'autre. Des mots comme «acte», «demande» ou «enfant», par exemple, une fois qualifiés : «acte authentique », "demande reconventionnelle» ou «enfant légitime» appartiennent clairement au vocabulaire juridique. Par quel système combinatoire en est-on arrivé à cette certitude ? La difficulté réside dans la détermination du moment où la langue courante devient spécialisée.

Certains chercheurs pensent que la langue de spécialité et la langue courante sont distinctes, que celle-là exclut les éléments propres à celle-ci. À la limite, on peut concevoir que certains vocabulaires très spécialisés, voire certaines structures ou tournures particulières à un domaine ne peuvent être assimilés à ce que l'on qualifie commodément de «langue courante» ${ }^{7}$. Il s'agirait

5. Et juridiques. Rappelons que certains théoriciens, dont Piaget, ne rangent pas le droit parmi les sciences nomothétiques, celles qui cherchent à dégager des lois ou des relations quantitatives comme la linguistique, l'économie ou la démographie ont vocation de le faire.

6. Les adjectifs varient selon les valeurs d'emploi (langue «courante», "usuelle», "commune», "vulgaire», "ordinaire»...). L'expression retenue est la plus généralement employée (cf. Petit Robert (1977): 1, 2e éd., p. XVII) comme marque d'usage.

7. Voir sur cette question J.-L. Sourioux et P. Lerat (1975): Le langage du droit, Paris, P.U.F., et Georges Legault (1977) : La structure performative du langage juridique, Montréal, P.U.M.' 
donc d'exceptions dont on ne saurait tirer des lois générales. D'autres chercheurs, les plus nombreux, pensent avec B. Malmberg qu'il est impossible de tracer une «limite absolue entre langue courante et langue spécialisée ${ }^{8}$.

Les terminologues, en distinguant dans le champ d'investigation les niveaux notionnel et fonctionnel, impriment un tour concret à la notion de langue de spécialité. La méthode d'analyse contextuelle notamment, que propose Robert Dubuc ${ }^{9}$, répond davantage, semble-t-il, aux besoins spécifiques des traducteurs, terminologues et rédacteurs qui étudient plutôt le langage écrit, particulièrement en situation comparative, celle qui nous intéresse ici. En relevant un certain nombre de descripteurs significatifs, le terminologue arrive généralement à remonter à la source notionnelle ou fonctionnelle de l'unité terminologique spécialisée.

Toutefois, cette démarche ponctuelle ne prend en considération que certains éléments de ce qu'il est convenu d'appeler le discours ${ }^{10}$, c'est-à-dire le sens global de l'énoncé. La dichotomie introduite par Darbelnet ${ }^{11}$ entre nomenclature et vocabulaire de soutien éclaire le discours juridique. Le terme technique, à lui seul, n'est qu'un micro-élément du discours spécialisé qui ne revêtirait sa pleine signification qu'encadré par le vocabulaire de soutien. À titre d'exemple, des mots neutres tels que «public», "positif» et «anonyme» confèrent toute leur valeur aux expressions «ordre public», «droit positif» et «société anonyme».

Nous pouvons dès lors poser en principe que la notion de langue de spécialité est fonction d'un groupe donné de variables selon le domaine, le type de public et le genre de besoins considérés. Il est par conséquent nécessaire de l'adapter aux conditions particulières d'un enseignement axé sur la langue juridique, dans le cadre d'un programme de traduction par exemple.

\section{Spécificité de $L S$}

Si l'on tient compte des contraintes propres à l'enseignement des langues de spécialité en contexte bilingue, que l'on parte de l'anglais ou du français, ou qu'il s'agisse d'un enseignement se limitant à l'une de ces deux langues, la plupart des définitions proposées n'expriment qu'un minimum de variables pouvant convenir. C'est à une démarche sémasiologique qu'il faut faire appel pour qualifier la langue juridique puisqu'il est nécessaire de partir des signifiants de l'énoncé (ou du texte) d'origine pour aller à la signification du concept donné.

La notion de langue juridique ne se réduit pas à un simple exercice de terminologie et de style, selon la distinction classique entre fond et forme. Un troisième élément, celui de la structure du langage, vient s'interposer entre le contenu purement notionnel et la forme purement syntaxique, les reliant entre eux et leur conférant une homogénéité, une spécificité propres qui caractérisent les discours administratif, commercial, scientifique, juridique, etc.

8. Actes du Stage de Saint-Cloud (1971): Paris, Didier, p. 91.

9. Manuel pratique de terminologie (1978): Montréal, Linguatech. 10. Lire sur cette question l'article de J. Darbelnet (1979): «Réflexions sur le discours juridique »,
Meta, vol. $24, \mathrm{n}^{\circ}$ 1, p. 26-34.

11. Ibid., p. 26 


\section{Obstacles inhérents aux sciences juridiques}

Toutefois, quatre handicaps freinent l'évolution méthodologique de la langue de spécialité juridique: la définition du droit, le langage du droit, le conflit des méthodes et les problèmes de classification.

\section{L'objet du droit}

Nul ne saurait enseigner une matière «scientifique» sans en avoir au préalable clairement défini l'objet. Peut-on reconnaître, dans ces conditions, le statut de science à une discipline dont on saisit mal ce vers quoi elle tend? Étant donné que théoriciens et praticiens divergent sur la nature exacte de leur art, la stabilité sémantique qui caractérise les sciences dites exactes ne s'applique pas ou très mal dans le cas du droit. Il suffit de se livrer à une rapide étude comparée des trois ou quatre principaux systèmes juridiques et d'en analyser la fin pour s'en convaincre. La question de la définition amène naturellement celle du langage.

\section{Le langage de Thémis}

De toutes les langues spécialisées, la langue juridique est peut-être celle où règne la plus grande polysémie, au point que Gény a pu dire que «la technique juridique aboutit, pour la plus grande part, à une question de terminologie ${ }^{12}$. Tel est certainement le cas en régime de common law où l'interprétation du langage complexe et parfois ambigu des lois est à la source de nombreux conflits ${ }^{13}$. L'incertitude sémantique qui caractérise le langage du droit et l'absence d'un fonds terminologique essentiel ${ }^{14}$ en rendent particulièrement délicate son étude. La méthodologie de cette langue de spécialité reflète ces incertitudes.

\section{Les conflits de méthodes}

De plus, les méthodes d'étude du droit sont en perpétuelle évolution. Cela ne peut que se refiéter sur l'étude de son langage. Depuis des siècles, juristes et scientifiques s'opposent sur le sens et la portée des méthodes d'établissement des faits. Par ailleurs, chez les juristes mêmes l'unanimité est loin d'être faite. Pour les uns, la "méthode des cas" est érigée en système, les précédents de jurisprudence (principe du stare decisis) font plus ou moins obligation au juge, selon les pays, de suivre la règle dégagée par d'autres juges. Pour les autres, la jurisprudence joue un rôle mineur et la loi (le «Code») est l'autorité souveraine. Peut-on affirmer qu'une méthode est supérieure à l'autre? En vertu de quels principes? Les réformes qui sont régulièrement apportées aux différents programmes des facultés de droit les plus prestigieuses démontrent la vanité d'une telle hypothèse: il n'existe pas de méthode idéale. Cela m'amène à poser le problème de la classification.

12. François Gény (1921): Science et technique en droit privé positif, Paris, Sirey, t. III, $\mathbf{n}^{\circ} 255$, p. 456.

13. Voir sur cette question Elliott Biskind (1975) : Simplify Legal Writing, New York, Arco, p. 4.

14. Voir Meta, vol. $24, n^{\circ} 1$, p. 41-44. 


\section{Conflits de classification}

Quiconque aborde l'étude de la langue de spécialité juridique en perspective comparatiste est frappé par les différences que présentent les méthodes de classification d'un système à l'autre. Aux grandes divisions générales et, dit-on, logiques des systèmes civilistes, certains systèmes, dont celui de la common law, préfèrent recourir à des distinctions pratiques et concrètes fondées sur la procédure.

Le second type d'obstacles procède des différences entre les deux systèmes juridiques.

\section{Obstacles présentés par l'étude comparative de deux systèmes juridiques}

En théorie, la comparaison de deux systèmes juridiques pose de nombreux problèmes de fond puisqu'il n'existe pas deux systèmes rigoureusement semblables. Cela ne porte toutefois pas à conséquence lorsqu'on ne vise qu'à informer de futurs spécialistes destinés à travailler dans un seul système. Dans la pratique, il en va différemment lorsque ces mêmes spécialistes sont appelés à travailler (en l'occurrence à traduire) à cheval sur deux systèmes. Cette hypothèse appelle trois remarques: des institutions différentes impriment aux concepts qu'elles dégagent une relativité qui se traduit par la difficulté que pose toute tentative de normalisation aux terminologues et aux lexicographes.

\section{Des institutions différentes}

Un système juridique repose sur un édifice social et politique répondant aux aspirations, aux us et coutumes d'un peuple. Cet édifice est rarement comparable à celui d'un autre pays, même si les origines de leurs systèmes politiques ont des points communs. Montesquieu avait déjà fait observer que «c'est un très grand hasard si celles (les lois politiques et civiles) d'une nation peuvent convenir à une autre ${ }^{15}$. Cette constatation est toujours actuelle. Il en découle une relativité certaine des concepts.

\section{Relativité des concepts}

Contrairement à ce que l'on peut observer dans les sciences exactes, les concepts juridiques présentent une certaine instabilité et se prêtent parfois mal à l'analyse. Ce phénomène est amplifié par la diversité des systèmes en présence. Tel concept de LD peut ne pas avoir de correspondance dans LA. Pis, telle notion existe dans les deux systèmes mais ne recouvre pas les mêmes réalités. Ceci pose le problème de la documentation et, plus particulièrement, de la lexicographie juridique.

\section{Le problème de la documentation}

L'absence d'une documentation fiable et relativement complète est criante dans le domaine des sciences juridiques. Peu de pays possèdent des diction- 
naires juridiques répondant aux normes lexicographiques des grandes productions comme le Littré, l'Oxford Dictionary, le Robert ou le Webster. Et quand ils existent, ils sont difficilement exportables puisque la terminologie employée dans un pays est rarement utilisable dans un autre. L'exemple du Québec est édifiant: la plupart des principes regroupés dans le Code civil de la Province de Québec ont une résonance française bien légitime car ils s'inspirent directement du Code Napoléon, même si certains d'entre eux trahissent l'influence du droit anglais, comme les «lois commerciales» (Livre quatrième) introduites dans un code «civil». En revanche, le Code criminel du Canada, rédigé en anglais, est traduit dans un français qui trahit son origine, et la classification des "common law crimes" en trois grandes catégories ${ }^{16}$ ne correspond que de très loin aux principes correspondants du Code pénal français ${ }^{17}$.

La spécificité des notions et de la terminologie juridiques constitue un des obstacles majeurs à l'élaboration d'une méthode globale de traduction juridique. La solution passe nécessairement par l'étude ponctuelle des situations intervenant dans le contexte des langues et des systèmes juridiques considérés.

\section{Éléments du discours juridique}

Je me bornerai aujourd'hui à une brève étude comparée de la langue et du style juridiques en prenant l'anglais et le français comme langues de référence.

On entend souvent parler de «langage du droit». Ce générique recouvre en réalité plusieurs langages particuliers qui forment une typologie essentielle quoique limitée, qu'il est nécessaire de rappeler pour saisir dans toute sa portée l'étendue et l'importance du discours juridique :

1. En premier lieu vient le langage de la Loi, le style législatif, qui incarne aux yeux de beaucoup l'essence même du discours juridique;

2. Je placerai en deuxième lieu le langage de la Justice, le discours judiciaire, et le style bien particulier que l'on attribue souvent au langage de Thémis;

3. En troisième lieu, je mettrai le langage "réglementaire", celui de l'Administration, des textes destinés au public; c'est en quelque sorte l'émanation du droit administratif, le style «administratif» proprement dit, celui que connaît le grand public (et qu'il assimile généralement à la «loi»);

4. Le langage juridique des affaires, celui du droit commercial, qui revêt tant de formes différentes, dont la plus connue est celle du contrat (de vente);

5. Le langage essentiellement «privé», celui du droit civil, qui peut prendre la forme d'un acte sous seing privé ou d'un testament, mais qui est, le plus souvent, caractérisé par le style «notarié»;

6. Le langage de la «doctrine» (des théoriciens du droit), qui peut, lui aussi, revêtir de multiples aspects: manuels de droit, articles juridiques, commentaires d'arrêt, avis juridique, etc.

16. Treason, Felony, Misdemeanour (1965) : Fundamentals of Canadian Law (by F.A.R. Chapman), Toronto, McGraw-Hill, p. 30.

17. Comparer l'article $1 \mathrm{er}$ du Code pénal français et l'article 294 du Code criminel canadien. 
Cette typologie n'est nullement exhaustive. Cette classification pourra même sembler arbitraire: on pourrait en effet s'inspirer du pragmatisme des méthodes anglo-saxonnes et prêter au langage du droit des manifestations découlant d'un autre ordre logique. Il reste que la Loi est le mode d'expression juridique universel et que le cadre de cet exposé ne me permet pas d'étendre mon étude aux diverses formes d'expression du langage du droit, ni d'analyser en profondeur certains problèmes que pose la traduction en français de textes de loi rédigés en anglais. Du moins est-il possible d'avancer quelques raisons, d'ordre pratique pour la plupart et bien connues des spécialistes, mais sur lesquelles il reste encore tant à dire avant d'atteindre le degré d'objectivité indispensable pour se livrer à une étude exempte des a priori habituels. En voici un exemple: le Code civil serait un modèle universel de clarté, de rigueur et de logique qui, depuis près de deux siècles, fait l'orgueil des juristes français voués au culte du Code Napoléon, dont le mythe est particulièrement vivace. En comparaison, la forme des lois anglaises serait l'exemple à ne pas suivre, révélateur du manque de clarté, de rigueur et de logique des légistes anglais!

De tels raccourcis n'ont pas leur place en linguistique car ils déforment une réalité extrêmement complexe en l'enfermant dans une formule par trop simpliste. En droit, comme dans les autres sciences humaines et sociales, rien n'est définitif, figé. Vouloir comparer les styles législatifs anglais et français reviendrait à essayer de comparer deux auteurs, un classique et un romantique, Boileau et Chateaubriand (ou Victor Hugo), La Fontaine et l'auteur de Vanity Fair...

On devrait plutôt tenir compte des différences, des besoins propres à chaque système tout en respectant le parallélisme des textes en présence, c'està-dire éviter autant que possible de dérouter le lecteur en attribuant le numéro 32 , dans la version française, à l'article qui porte le numéro 27 dans le texte anglais de départ, sous prétexte que la «logique» ou le "génie» de la langue française font obligation de bouleverser l'ordonnance établie par le législateur... $\mathrm{Si}$ de tels principes devaient inspirer le traducteur, personne ne serait en mesure de lire l'original et ses traductions dans les 4, 5 ou 6 langues officielles que comptent les organismes internationaux!

Pour revenir à des considérations plus réalistes, voyons en quoi, outre la forme même de la rédaction, les deux langues et les deux styles diffèrent.

\section{Remarques d'ordre lexical}

Comme il serait trop long d'entrer dans le détail du développement historique des deux systèmes considérés, je me bornerai à répéter que, contrairement à certaines idées reçues, ils ont des points en commun, ne serait-ce que la présence d'un grand nombre de termes venant du latin, auquel on doit de nombreux archaïsmes. La langue latine a beaucoup marqué l'anglais juridique, et la langue anglaise en général ${ }^{18}$. Quel traducteur n'a pas rencontré, au hasard des textes, de savoureuses locutions latines comme res ipsa loquitur, in pari

18. Voir particulièrement David Mellinkoff (1973): The Language of the Law, Boston and Toronto, Little, Brown and Company, p. 13. 
delicto, nolo contendere ou corpus delicti? Ces rencontres, quoique moins fréquentes, ne sont pas rares dans la langue juridique française. L'emploi du latin est néanmoins plus rare dans les textes de loi $^{19}$, surtout les plus récents.

Un autre élément connu de la langue juridique anglaise est la grande place qu'y tiennent les mots d'origine française, reliquat de quatre cents ans de primauté du français en Angleterre. Si la plupart des traducteurs connaissent l'origine de mots tels que action, assault, demand, justice, obligation, etc., qui ont été empruntés directement au français de l'époque, seuls quelques philologues avertis savent l'origine française de mots comme:

- purchase: du vieux français «purchacen» ou «porchacier» = pourchasser;

— chattels : du vieux français «chatel » (à rapprocher de cattle; ces deux mots tirent d'ailleurs leur origine de l'adjectif latin capitalis, $e$ ) = bien(s) mobilier(s);

- larceny : du moyen français «larcin»;

-attorney: du moyen français "atorné», du verbe «atourner»= diriger, disposer.

Je pourrais allonger la liste à loisir ; pour cela il suffirait de relever systématiquement les termes juridiques contenus dans l'Oxford Dictionary ou le Webster ${ }^{20}$. Mais il est d'autres problèmes que lexicaux; en outre, le traducteur est averti de longue date des tours que peuvent lui jouer les «faux amis». Je cite pour mémoire :

- agreement : accord, convention et non «agrément» (= consent)

- battery : voies de fait (assault and battery = coups et blessures) et non «batterie »

- count : chef (d'accusation) et non «compte»

- evidence : preuve (et non «évidence»)

- felony : selon les pays (G.-B., É.-U.), délit particulièrement grave ( \pm crime)

- partner : associé (et non partenaire)

- reasons : motifs (d'une décision) et non les «raisons"

etc. Il sait que des mots empruntés par une langue entre les XIt et XVIe siècles ne peuvent avoir conservé leur sens original quelque... neuf cents ans plus tard.

\section{Remarques d'ordre syntaxique}

La syntaxe me semble poser un problème d'une tout autre gravité. Prenons un exemple classique entre tous, celui de l'emploi des temps, source de nombreux ennuis chez le traducteur.

Le maniement des temps du passé (preterite = imparfait, passé composé ou passé simple; present perfect $=$ présent indica.) ou même du présent est loin d'être acquis. Trop souvent shall est pris pour un futur quand il n'a que valeur emphatique.

Exemple: There shall be a legal committee $=$ Un comité juridique est institué (et non «doit être institué» ou «sera institué»).

Pour la plupart des linguistes nord-américains, shall avec valeur de futur est un archaïsme. Il abonde pourtant dans les textes de loi sous sa forme emphatique et on l'emploie même pour exprimer des modalités qui ne sont pas toujours d'ordre obligatoire ou contraignant.

19. I] ne faudrait pas en conclure que le latin est désormais banni des textes juridiques. Cette tendance découle du désintérêt général pour une discipline jugée «inutile», que l'on observe dans la plupart des nations traditionnellement prolatinistes.

20. Voir également David Mellinkoff, op. cit., p. 15. 
Exemple : «This Act shall apply...» = La présente Loi s'applique... (et non «doit s'appliquer»)

"The Director shall be entitled...» = Le Directeur peut... (et non «doit» ou «pourra»)

La difficulté consiste à distinguer la forme comminatoire

"A corporation shall, not later than seven days..." = La société doit, dans les sept jours... ${ }^{21}$

— «The Director shall forthwith send a copy...»= Le Directeur doit immédiatement envoyer un exemplaire... 22

de la forme «neutre» ou simplement emphatique

— No person shall be appointed as trustee...» $=/ \ldots /$ une personne ne peut être nommée fiduciaire ${ }^{23}$.

- He shall be guilty = il (le prévenu) est coupable.

Les deux langues diffèrent donc considérablement sur certains plans syntaxiques, bien que cette particularité ne soit pas caractéristique du langage du droit. Le même genre d'observation pourrait être fait entre l'espagnol (ou l'allemand, l'italien...) et le français. Il est cependant un autre point de divergence important entre l'anglais et le français juridiques, c'est l'aspect proprement stylistique.

\section{Remarques d'ordre stylistique}

Les lois de deux nations, de deux sociétés différentes ne diffèrent pas seulement sur le fond, dans l'esprit, elles s'opposent également dans la lettre.

Les principes d'exposition et de rédaction peuvent varier considérablement d'un pays et d'une culture à l'autre, parfois au sein d'une même nation, et confèrent au style législatif une spécificité qui lui est propre. On a coutume d'opposer avec quelque raison le style de la common law et le style civiliste comme l'exemple classique de deux conceptions diamétralement opposées. Sans entrer dans les détails, je vais étayer cette allégation par quelques exemples.

Je vous ferai grâce de la lecture d'extraits de la Loi de l'impôt sur le revenu des États-Unis ${ }^{24}$ et me contenterai de citer deux articles du Code civil, à titre d'exemple. L'article 2, un des plus célèbres, tout d'abord :

«La Loi ne dispose que pour l'avenir; elle n'a point d'effet rétroactif.»

et l'article 6 :

"On ne peut déroger, par des conventions particulières, aux lois qui intéressent l'ordre public et les bonnes mœurs. »

C'est clair, net et concis. C'est malheureusement un idéal qui appartient au passé quand on sait ce qu'il est advenu de la rédaction législative française depuis, en gros, la seconde guerre mondiale ${ }^{2 s}$ : elle n'a cessé de se dégrader

21. Article 184. (12), Canada Business Corporations Act, 1974-75-76, c. 33 amended by 1976-77, c. $52,1978-79$, c. 9 , déc. 1978.

22. Article 181. (5), ibid.

23. Article 78. (1), ibid.

24. Qui passe pour ce que l'on peut concevoir de pire en la matière. L'équivalent canadien ne vaut, hélas, guère mieux.

25. Selon certains spécialistes, l'origine du mal remonterait au Traité de Versailles, signé en 1919, quand l'influence des juristes américains a commencé à s'exercer sur leurs homologues européens. 
pour céder à l'inflation verbale, à la redondance et à l'imprécision dénoncées notamment par Robert Catherine ${ }^{26}$. Ce sont là les défauts que les civilistes attribuent avec complaisance à leurs homologues anglais, américains et canadiens. C'est l'histoire de la paille et de la poutre...

En simplifiant vraiment beaucoup les choses, je dirai que le style législatif français répond (dans l'idéal théorique) à deux principes cardinaux : la règle générale posée en principe et la concision, soit dire le plus avec le moins de mots possible, tout en restant précis.

Qu'observe-t-on en common law? Exactement l'inverse: au lieu des principes généraux, la loi expose une multitude de détails qui peuvent sembler déplacés. En outre, elle commence toujours par une longue liste de définitions de la plupart des termes contenus dans le texte, afin d'éviter les erreurs d'interprétation. Cela peut donner 10 pages de définitions comme dans le Code criminel. Il est difficile de concevoir deux démarches plus différentes. Elles posent de gros problèmes au traducteur, par ailleurs tenu de respecter l'ordre alphabétique français, ce qui oblige le lecteur à une incessante gymnastique de recherche du terme voulu.

$$
\begin{aligned}
\text { Exemple }: \text { corporation } & =\text { société } \\
\text { auditor } & =\text { vérificateur } \\
\text { individual } & =\text { particulier }
\end{aligned}
$$

Ensuite, étant donné qu'en français la loi parle au présent (ce qui exclut l'emploi du futur puisque la règle énoncée est d'application immédiate), que le masculin comprend le féminin (par souci de précision et d'économie : plus une phrase est longue, plus elle perd de sa rigueur, de sa précision et de sa clarté) et que le singulier comprend le pluriel, on voit d'emblée le genre de difficultés que doit surmonter le traducteur qui, partant d'un texte très long et détaillé à l'extrême, devrait produire exactement l'inverse : un texte concis, de portée générale. La tâche est quasi impossible. Reste la solution du compromis. Il le faut bien, pour rendre la traduction fonctionnelle dans le contexte juridique canadien: la version française des textes fédéraux canadiens reflète cette réalité. En revanche, le Québec peut se permettre, avec la loi 101, d'innover en l'espèce et d'opter pour une rédaction législative résolument française au point d'être érigée en modèle.

Faute de temps et de place, je ne parlerai pas des nombreuses autres particularités des deux styles en présence. Il y aurait encore beaucoup à dire sur l'énonciation juridique, les procédés propres à chaque système linguistique, entre autres sur les coordonnées déictiques de l'anglais et du français, les anaphores et les anastrophes, etc.

\section{Conclusion}

En résumé, malgré les origines communes et les universaux linguistiques qui unissent la plupart des grandes langues occidentales, le fossé qui sépare les principes de la rédaction législative, en régime de common law et en régime civiliste, $\mathbf{n}$ 'est pas près d'être comblé. Les conceptions, les mentalités sont en-

26. Robert Catherine (1968) : Le style administratif, Paris, Albin Michel. 
core très ancrées dans un passé récent que seul le temps pourra oblitérer. Au rythme des relations internationales, peu à peu il se dégage par osmose des principes «communautaires» sur lesquels finiront par se rejoindre les grandes traditions et les grands systèmes juridiques pour former l'union symbiotique déjà réalisée dans quelques domaines ${ }^{27}$. Fera-t-elle le bonheur du traducteur? Du train où vont les choses, ce dernier a tout loisir de s'y préparer.

$\overline{27 . ~ I n t e r p o l, ~ " L ' e s p a c e ~ j u d i c i a i r e ~ e ́ u r o p e ́ e n ~ » ~(C o m m u n a u t e ́ ~ e ́ c o n o m i q u e ~ e u r o p e ́ e n n e), ~ p a r ~ e x e m p l e . ~}$ 